\title{
Hyperbolic reductions for Einstein's equations
}

\author{
Helmut Friedrich \\ Albert Einstein Institut, Max Planck Institut für Gravitationsphysik, Schlaatzweg 1, 14473 \\ Potsdam, Germany \\ Received 26 January 1996, in final form 2 April 1996
}

\begin{abstract}
We consider the problem of reducing initial value problems for Einstein's field equations to initial value problems for hyperbolic systems, a problem of importance for numerical as well as analytical investigations of gravitational fields. The main steps and the most important objectives in designing hyperbolic reductions are discussed. Various reductions which have already been studied in the literature or which can easily be derived from previous discussions of the field equations are pointed out and some of their specific features are indicated. We propose new reductions based on the use of the Bianchi equation for the conformal Weyl tensor. These reductions involve symmetric hyperbolic systems of propagation equations and allow a number of different gauge conditions. They use unknowns in a most economic way, supplying direct and non-redundant information about the geometry of the time slicing and the four-dimensional spacetime. Some of this information is directly related to concepts of gravitational radiation. All these reductions can be extended to include the conformal field equations. Those which are based on the ADM representation of the metric can be rewritten in flux conserving form.
\end{abstract}

PACS numbers: 0420C, 0420E

\section{Introduction}

In accordance with the basic tenets of general relativity, Einstein's field equations should imply causal propagation of the gravitational field and should be hyperbolic. Because of their covariance, however, the equations are not hyperbolic in an immediate sense. The derivation of general statements about the existence, uniqueness and stability of solutions by analytical methods requires as an extra step the conversion of geometric initial value problems for Einstein's field equations into Cauchy problems for hyperbolic differential systems. This process, referred to in the following as 'hyperbolic reduction', is the subject of this paper.

While a number of hyperbolic reductions have been considered for some time in analytical studies, they have so far played only a minor role in numerical relativity. One can start finite differencing with equations which are not manifestly hyperbolic. However, since more and more complicated and, in particular, genuinely four-dimensional problems are being attacked, there is a growing interest among numerical relativists in using hyperbolic propagation equations.

Hyperbolicity has strong implications for the nature of the characteristics and thus the propagational properties of the equations. This is reflected in the Courant-Lewy-Friedrichs condition and similar requirements known for a long time in numerical analysis of hyperbolic field equations.

In general relativity, as compared with other theories based on hyperbolic field equations, the structure of the characteristics plays an even more central role because it is closely 
related to the basic field of the theory. Suitable hyperbolic reductions should ensure causal propagation. The characteristics should be timelike or null and the outer sheet of the cone of characteristic rays should coincide with the null cone of the gravitational field. Since the null cone determines the metric field up to conformal rescalings, almost equivalent concepts are denoted by 'causal structure', 'conformal structure' and 'structure of characteristics'. They are distinguished mainly by emphasizing different points of view.

Since in general relativity the analysis of the causal structure has proven so important in local as well as in global studies and since hyperbolicity is intimately related to causality, it seems advisable also to make this relationship in numerical investigations manifest in the form of the equations. The necessity of this becomes particularly obvious in calculations near lightlike horizons. In this situation a subtle interplay occurs between the causal structure of the solution, the analytical characteristics of the equations, and the "numerical characteristics' which depend on the equations singled out by the hyperbolic reduction. Furthermore, gauge conditions involving acausal propagation of gauge-dependent quantities may lead to numerical as well as interpretational difficulties of various sorts.

One reason for the recent interest in hyperbolic reductions is the desire to utilize the expertise gathered in fields which have a longer tradition of numerical investigations. It remains to be seen to what extent methods which have been developed according to the particular needs and phenomena observed, e.g. in hydrodynamics, can be transferred successfully to relativity. Perhaps it will prove more important to copy the long tradition in hydrodynamics of relating analytical investigations to numerical ones. Furthermore, it is desirable to convert, if possible, interesting numerical findings, which indicate new phenomena of a general nature, into analytical theorems. The recent tendency of numerical relativists to use hyperbolic systems should simplify this task since these systems are more amenable to analytic discussion.

To give a survey of hyperbolic reductions which is general enough to offer the optimal reduction in any given situation appears a hopeless task. There are various concepts of hyperbolicity available in the literature and it may be good to keep this in mind while seeking for a reduction designed to satisfy specific needs. However, no attempt will be made here at presenting hyperbolicity in any generality. Instead I shall discuss in section 2 some known examples of hyperbolic reductions, various aspects of the general idea, and some of the most important objectives in the construction of hyperbolic reductions. In sections 5 and 6 some new examples of reductions using symmetric hyperbolic systems will be introduced. These examples employ different representations of the metric but all of them use the Bianchi identity, discussed in some detail in section 4, as a basic ingredient.

The importance of the Bianchi identity has been documented in various analytical investigations. Most interesting for the present discussion is the fact that irrespective of the gauge this identity implies hyperbolic propagation equations for the conformal Weyl tensor. Because of its invariant nature the Bianchi identity offers a rich choice of possibilities to perform reductions which, moreover, can be well adapted to various geometrical situations and allow us also to deal with situations where source fields are present. Finally, the reduction procedures indicated here extend easily to the case of the conformal field equations which allow numerical calculations of infinite spacetimes on finite grids.

How well the hyperbolic systems proposed in this and other articles behave in numerical calculations is a question which cannot be discussed at present. This is not only due to the ignorance of the present author but also to the general lack of experience with all these systems in numerical calculations under fairly general assumptions. However, because of its invariant nature, its enormous flexibility, and its behaviour under conformal rescalings it may be expected that the Bianchi identity will prove useful in numerical studies to the 
same extent it has proved useful in analytical investigations.

\section{General remarks on reduction procedures}

In the following we shall only consider the vacuum field equations since their analysis usually extends easily to the more general case where source fields are present. To compare different hyperbolic reductions and to speak about them in a general way, it will be convenient to introduce a few basic concepts and illustrate them by discussing a specific example. A reduction procedure consists essentially of four steps.

(i) Choice of the representation of the Einstein equations and of the basic unknowns. For the sake of illustration we shall consider in the following the system of metric coefficients $g_{\mu \nu}$ in some coordinates as the basic unknowns and the Ricci tensor, considered as a secondorder operator acting on $g_{\mu \nu}$, as the basic operator such that the vacuum field equations take the form

$0=R_{\mu \nu} \equiv-\frac{1}{2} g^{\alpha \beta}\left\{\frac{\partial^{2} g_{\mu \nu}}{\partial x^{\alpha} \partial x^{\beta}}+\frac{\partial^{2} g_{\alpha \beta}}{\partial x^{\mu} \partial x^{\nu}}-\frac{\partial^{2} g_{\mu \beta}}{\partial x^{\alpha} \partial x^{\nu}}-\frac{\partial^{2} g_{\alpha \nu}}{\partial x^{\mu} \partial x^{\beta}}\right\}+H_{\mu \nu}^{\prime}(g, \partial g)$

with a certain function $H_{\mu \nu}^{\prime}$ of the metric coefficients and their derivatives. As it stands the equation is not hyperbolic in any known sense.

(ii) Choice of the gauge and of the 'coordinate gauge source functions'. Given the representation of the field equations above, a possible way of identifying a useful gauge is to introduce the quantity $\Gamma^{\mu}=g^{\alpha \beta} \Gamma_{\alpha}{ }^{\mu}{ }_{\beta}$, with $\Gamma_{\nu}{ }_{\nu}{ }_{\rho}$ denoting the Christoffel symbols of $g_{\mu \nu}$, and to rewrite the equation above in the form $[6,23]$

$$
0=-\frac{1}{2} g^{\alpha \beta} \frac{\partial^{2} g_{\mu \nu}}{\partial x^{\alpha} \partial x^{\beta}}+g_{\alpha(\mu} \nabla_{\nu)} \Gamma^{\alpha}+H_{\mu \nu}(g, \partial g),
$$

where we consider $\nabla_{\nu} \Gamma^{\mu}$ to be given by the expression which we would obtain if the $\Gamma^{\mu}$ were components of a vector field. If the functions $\Gamma^{\mu}$ were known in this equation, we would have a system of wave equations, i.e. the prototype of a hyperbolic system. Two interesting observations can be made concerning these functions. Firstly, for given coordinates $x^{\mu}$, the functions $\Gamma^{\mu}$ are obtained by applying the invariant wave operator to the coordinate functions, i.e. $\Gamma^{\mu}=-\nabla_{\rho} \nabla^{\rho} x^{\mu}$. Secondly, for given smooth functions $f^{\mu^{\prime}}=f^{\mu^{\prime}}\left(x^{\lambda^{\prime}}\right), \mu^{\prime}=0,1,2,3$, defined for $x^{\lambda^{\prime}}$ in some open subset of $\mathbb{R}^{4}$, we may consider the system of semi-linear wave equations $\nabla_{\rho} \nabla^{\rho} x^{\mu^{\prime}}=-f^{\mu^{\prime}}\left(x^{\lambda^{\prime}}\right)$ for unknown functions $x^{\mu^{\prime}}$. Solving these equations for Cauchy data on a spacelike hypersurface such that the differentials $\mathrm{d} x^{\mu^{\prime}}$ are independent there, we obtain a local coordinate system. If the Christoffel symbols are expressed in these coordinates we get $\Gamma^{\mu^{\prime}}=f^{\mu^{\prime}}$. Thus, by suitable choice of coordinates the functions $\Gamma^{\mu}$ can locally be given any preassigned form and conversely they determine the coordinates via wave equations from suitably given initial data. We call functions serving such purposes 'gauge source functions'.

(iii) Preparation of the 'reduced initial value problem'. In the example above we choose coordinate gauge source functions $f^{\mu}$ and consider the 'reduced equations'

$$
0=-\frac{1}{2} g^{\alpha \beta} \frac{\partial^{2} g_{\mu \nu}}{\partial x^{\alpha} \partial x^{\beta}}+g_{\alpha(\mu} \nabla_{\nu)} f^{\alpha}+H_{\mu \nu}(g, \partial g)
$$


as propagation equations for the metric coefficients. On the initial hypersurface $S=\left\{x^{0}=\right.$ $0\}$, which is assumed to be spacelike, let $h, \chi$ be geometrical initial data satisfying the constraints on $S$ (cf [2] and section 6). Thus the Riemannian metric $h$ and the symmetric tensor field $\chi$ are, respectively, assumed to represent the first and the second fundamental form induced on $S$ by the metric $g_{\mu \nu}$ which we want to construct. By suitable choice of the coordinates and their differentials on the initial hypersurface, we prepare the initial data $g_{\mu \nu}, \partial_{0} g_{\mu \nu}$ derived from $h, \chi$ such that $\Gamma^{\mu}(g, \partial g)=f^{\mu}$ on $S$. It may be noted that there are several ways of doing this, but in any case we now have a well posed initial value problem which has a unique smooth solution $g_{\mu \nu}$ on a certain domain containing $S$.

(iv) Demonstration that the solution to the reduced problem does satisfy the Einstein equations. From the solution $g_{\mu \nu}$ considered above, we derive the functions $\Gamma^{\mu}$. To see that we have in fact obtained a solution to the Einstein equations we need to show that $\Gamma^{\mu}=f^{\mu}$. Using the twice contracted Bianchi identity and (2.1) we derive the 'subsidiary equation'

$$
0=\nabla_{\rho} \nabla^{\rho}\left(\Gamma^{\mu}-f^{\mu}\right)+R_{\rho}^{\mu}\left(\Gamma^{\rho}-f^{\rho}\right) .
$$

By the way we set up the reduced initial value problem and from the fact that $g_{\mu \nu}$ solves the reduced equations we can deduce that the Cauchy data for $\Gamma^{\mu}-f^{\mu}$ on the initial hypersurface vanish. Since the subsidiary equations are wave equations, which possess the uniqueness property, we conclude that $\Gamma^{\mu}=f^{\mu}$ in a certain neighbourhood of the initial hypersurface.

The reduction argument above with the choice $\Gamma^{\mu}=0$, i.e. for 'harmonic coordinates', was discovered by Choquet-Bruhat [10]. The case of general gauge source functions has been discussed in [13].

Once the gauge source functions have been chosen, it is obvious what has to be done in step (iii); for further illustration see section 6.1.

Step (iv) may take different forms. While in our example above it needs to be shown that the gauge conditions propagate, the gauge is expressed explicitly in some of the examples considered later on by the form of the unknowns and it has to be shown that the constraints are preserved in the evolution of the data by the reduced equations (cf section 6.1). This step provides the only possibility of ensuring that a given choice of gauge source functions is consistent with the field equations. Most likely, progress in numerical calculations will rely on more and more intricate choices of gauge conditions. Therefore step (iv) needs to be performed to ensure the soundness of the procedure.

The related steps (i) and (ii) are decisive for any reduction. The first requirement is, of course, that the prospective gauge condition can be imposed locally without any restriction of generality and that it leads to hyperbolic reduced equations. However, the decision here will depend on considerations which go beyond the question of hyperbolicity.

We shall aim at a geometrical setting and a set of unknowns which allow an immediate and clear cut physical or geometrical interpretation of the results of numerical calculations. The unknowns should supply invariant information on the four-dimensional spacetime, possibly related to concepts of radiation and other notions of physical interest, as well as information on the nature of the three-dimensional time slices which are constructed in the numerical evolution.

We may want to select a set of gauge source functions which ensure the time slicing exists globally, i.e. in the maximal domain of dependence of the data, and which also entail nice behaviour of the time slices near horizons and at infinity. If there were an optimal 
time slicing for a given solution, it could in principle be characterized in terms of the gauge source functions given above. In fact, this is not possible since the slicing itself will depend on the evolution and cannot be characterized in a solution-independent local way. Ideally one would like to follow the numerical evolution of the spacetime and steer the propagation of the time slicing by a suitable adjustment of the gauge source functions in dependence on the geometry of the evolved spacetime; this poses several problems. The question of how the time slices react to changes in the gauge source functions has hardly been studied. Secondly, care must be taken not to introduce undue couplings into the equations. Furthermore, in the example considered above, the reduced equations are likely to change their type if the gauge source functions $f^{\mu}$ are allowed to depend on the derivatives of the metric coefficients.

There is an interesting case where the gauge source functions depend on the metric. This gauge condition essentially removes the freedom to perform diffeomorphisms, but it leaves us the freedom to choose coordinates as they appear suitable for our investigations. Assume that on the manifold $M$, on which we expect the solution $g_{\mu \nu}$ to exist, is given a symmetric connection which has connection coefficients $\gamma_{\mu}{ }^{\nu}{ }_{\rho}$ in some coordinate system. We require that the gauge source functions satisfy in the same coordinates

$$
0=\Gamma^{\nu}-g^{\mu \rho} \gamma_{\mu}^{\nu} \rho
$$

With these gauge source functions we get hyperbolic reduced equations and it turns out that this gauge is consistent with the field equations, i.e. it is preserved under propagation. Being obtained by contraction with the metric from the difference of two connections, the quantity on the right-hand side of the equation above is a tensor. Thus the equation holds in any coordinate system and the gauge condition can be imposed on the whole manifold.

In the particular case where the coefficients $\gamma_{\mu}{ }^{\lambda}{ }_{v}$ are the Christoffel symbols of a Lorentz metric $k_{\mu \nu}$ on $M$, which we shall refer to in the following as the 'harmonic map gauge', the equation above can be interpreted as saying that the identity map $i d_{M}$ is a harmonic map from the Lorentz space $(M, g)$ onto the Lorentz space $(M, k)$. This property can also be expressed by either of the equivalent conditions

$$
\nabla^{\rho} k_{\rho \mu}-\frac{1}{2} \nabla_{\mu} k_{\rho}{ }^{\rho}=0, \quad \hat{\nabla}_{\nu} g^{\mu \nu}-\frac{1}{2} g^{\mu \lambda} g_{\rho \nu} \hat{\nabla}_{\lambda} g^{\rho \nu}=0
$$

where $\nabla$ is the Levi-Civita connection defined by the metric $g$, which is also used to raise indices, and $\hat{\nabla}$ denotes the Levi-Civita connection defined by the metric $k$. Such gauge conditions have been used and discussed, in some cases without being aware that one is dealing with harmonic maps, in [7, 8] (for which [19] should be consulted), and in $[14,15,20]$.

The behaviour of the field equations under conformal rescalings usually plays an important role in analytical investigations of the solutions in the large [4,14-16]. Thus one may wish to choose a representation of the Einstein equations which allows explicit use of their conformal properties.

Finally and most importantly, we wish to arrive at reduced equations which show a favourable behaviour in numerical calculations. This question can hardly be discussed without performing sufficiently general numerical calculations for the various reduced systems which have been proposed. In certain contexts equations of divergence or flux conservative type (see below) have proved useful in hydrodynamics. To what extent this form of the equations will be favourable in relativity remains to be seen. Nevertheless, the reductions proposed in section 6 offer reduced equations which can be written in a flux conserving form.

The hyperbolic systems discussed below will not be of wave equation type but will be 'symmetric hyperbolic systems' [17]. These systems are of the form $A^{\mu} \partial_{\mu} u+b=0$ 
where the unknown $u$ takes values in some $\mathbb{R}^{N}, b$ is an $\mathbb{R}^{N}$-valued function and the $A^{\mu}$ are $N \times N$-matrix-valued functions which depend on the coordinates and the unknown $u$. The basic requirement on the system is that the matrices $A^{\mu}$ are symmetric (Hermitian in the case of complex-valued unknowns), i.e. satisfy ${ }^{\mathrm{T}} \bar{A}^{\mu}=A^{\mu}$, and that there exists a covector field $\xi_{\mu}$ such that $A^{\mu} \xi_{\mu}$ is positive definite for all admissible values of $u$ and the coordinates. Sometimes a system is called symmetric hyperbolic if it can be brought into the form above by a simple transformation like multiplication of the system by a suitable matrix-valued function.

Symmetric hyperbolic systems were used for the first time in general relativity by Fischer and Marsden [9], who combined the fact that wave equations imply symmetric hyperbolic systems [5] with the reduction procedure in harmonic coordinates discussed above to get symmetric hyperbolic reduced equations for the 10 components of the metric coefficients and their 40 first-order derivatives. Their system can also be rewritten in the divergence or 'flux conserving' form $\partial_{\mu} F^{\mu}(x, u)+b^{\prime}(x, u)=0$, where $F^{\mu}(x, u)$ is a smooth function of the coordinates and the unknown. Of course, in this reduction the harmonic map gauge may also be employed.

\section{The basic equations}

Again we denote the Christoffel symbols associated with the metric $g_{\mu \nu}$ by $\Gamma_{\rho}{ }^{\mu}{ }_{\nu}$. The curvature tensor is then given by

$$
R_{\nu \lambda \rho}^{\mu}=\partial_{\lambda} \Gamma_{\rho}{ }_{\nu}{ }_{\nu}-\partial_{\rho} \Gamma_{\lambda}{ }_{\nu}{ }_{\nu}+\Gamma_{\lambda}{ }^{\mu}{ }_{\delta} \Gamma_{\rho}^{\delta}{ }_{\nu}-\Gamma_{\rho}{ }_{\delta}{ }_{\delta} \Gamma_{\lambda}^{\delta}{ }_{\nu} .
$$

It satisfies the contracted Bianchi identity

$$
\nabla_{\mu} R_{\nu \lambda \rho}^{\mu}=\nabla_{\lambda} R_{\nu \rho}-\nabla_{\rho} R_{\nu \lambda} .
$$

The vacuum Einstein equations $R_{v \rho}=0$ are equivalent to

$$
R^{\mu}{ }_{\nu \lambda \rho}=C^{\mu}{ }_{\nu \lambda \rho}
$$

where $C^{\mu}{ }_{\nu \lambda \rho}$ denotes the (trace-free) conformal Weyl tensor. They imply the vacuum Bianchi identity, referred to in the following as the 'Bianchi equation',

$$
\nabla_{\mu} C^{\mu \lambda \rho}=0 \text {. }
$$

We shall consider the conformal Weyl tensor as one of our basic unknowns and the system (3.3), (3.4) as our basic representation of the Einstein equations. Precisely which fields will be considered as unknowns in addition to the conformal Weyl tensor depends on the representation of the metric.

In the orthonormal frame formalism we shall consider as unknowns the fields

$$
e_{k}^{\mu}, \quad \Gamma_{k}^{i}, \quad C_{j k l}^{i} .
$$

Here the metric is given in terms of the coefficients $e_{k}^{\mu}, \mu, k=0,1,2,3$, of an orthonormal frame $e_{k}$ in some coordinate system $x^{\mu}$ such that $e_{k}=e^{\mu}{ }_{k} \partial_{\mu}, g\left(e_{j}, e_{k}\right)=\eta_{j k}$, and thus $g^{\mu \nu}=\eta^{j k} e^{\mu}{ }_{j} e^{\nu}{ }_{k}$. In accordance with the spinor formalism employed below we shall always assume the signature $\eta_{j k}=\operatorname{diag}(1,-1,-1,-1)$ if we deal with the orthonormal frame formalism. The $\Gamma_{k}{ }_{j}{ }_{j}$ are the connection coefficients in this frame such that $\nabla_{k} e_{j} \equiv \nabla_{e_{k}} e_{j}=\Gamma_{k}{ }_{j}^{i} e_{i}$, where $\nabla$ denotes the Levi-Civita connection for $g$, and the $C^{i}{ }_{j k l}$ are the components of the conformal Weyl tensor in the frame $e_{k}$. Using $\eta_{j k}$ to lower indices, the fact that the connection is metric is expressed by the condition $\Gamma_{i j k}=-\Gamma_{i k j}$. Without assuming any gauge condition we deal with 50 unknown functions. 
In terms of these fields the field equations take the form

$\left[e_{p}, e_{q}\right]-\left(\Gamma_{p}^{l} q-\Gamma_{q}^{l}\right) e_{l}=0$,

$e_{p}\left(\Gamma_{q j}{ }^{i}\right)-e_{q}\left(\Gamma_{p j}{ }^{i}\right)-\Gamma_{k}{ }^{i}\left(\Gamma_{p}{ }^{k} q-\Gamma_{q}{ }^{k}\right)+\Gamma_{p k}{ }_{k} \Gamma_{q}{ }^{k}{ }_{j}-\Gamma_{q k}{ }^{i} \Gamma_{p}{ }^{k}{ }_{j}=C^{i}{ }_{j p q}$,

$\nabla_{i} C^{i}{ }_{j k l}=0$.

The first of these equations expresses the fact that the connection $\nabla$ is torsion free and the remaining equations are rewritings in the frame formalism of (3.3) and (3.4), respectively.

In dealing with the ADM representation we assume as usual that the signature is $(-1,1,1,1$,$) , that t=x^{0}$, and that latin indices take values $1,2,3$ such that the $x^{i}$ are coordinates on the slices $S_{c}=\{t=c\}$ of constant 'time' $c$. In the ADM representation the metric takes the form

$$
g=-(N \mathrm{~d} t)^{2}+h_{i j}\left(S^{i} \mathrm{~d} t+\mathrm{d} x^{i}\right)\left(S^{j} \mathrm{~d} t+\mathrm{d} x^{j}\right) .
$$

In this expression the lapse function $N$ is assumed to be positive, the interior Riemannian metric $h$ induced on the time slice $S_{t}$ is given by its coefficients $h_{i j}$ in a natural basis $\partial_{j}$, and the shift vector field $S=S^{\mu} \partial_{\mu}$, which is tangent to the time slices, is given by its spatial components $S^{i}$ since $S^{0}=0$. We denote by $h^{i j}$ the contravariant form of $h$ such that $h^{i j} h_{j k}=\delta^{i}{ }_{k}$.

The (future-directed) unit normal to the time slices is given by $n^{\mu}=(1 / N)\left(\delta^{\mu}{ }_{0}-S^{\mu}\right)$, whence $n_{\mu}=-N \delta^{0}{ }_{\mu}$. We write $h^{\mu}{ }_{\nu}=g^{\mu}{ }_{\nu}+n^{\mu} n_{v}$ for the orthogonal projector onto the time slices, $h_{\mu \nu}=g_{\mu \nu}+n_{\mu} n_{\nu}$ for the four-dimensional representation of the interior metric on the time slices, and $h^{\mu \nu}=g^{\mu \nu}+n^{\mu} n^{\nu}$.

Besides the ADM representation of the metric we need the corresponding representation of the connection coefficients. We find

$$
\begin{aligned}
\Gamma_{\mu}^{\nu}{ }_{\rho}=n_{\mu} n^{\nu} n_{\rho} & \frac{n(N)}{N}+n_{\mu} a^{\nu} n_{\rho}-a_{\mu} n^{\nu} n_{\rho}-n_{\mu} n^{\nu} a_{\rho} \\
& +n_{\mu} n_{\rho} \frac{1}{N} S_{, \pi}^{\nu} n^{\pi}-n_{\mu} \frac{1}{N} S_{, \pi}^{\nu} h_{\rho}^{\pi}-n_{\rho} \frac{1}{N} S_{, \pi}^{\nu} h_{\mu}^{\pi} \\
& +n^{\nu} \chi_{\mu \rho}-n_{\mu} \chi_{\rho}^{\nu}-\chi_{\mu}{ }^{\nu} n_{\rho}+\gamma_{\mu}{ }^{\nu}{ }_{\rho} .
\end{aligned}
$$

Here we use $n(N)=n^{\mu} \partial_{\mu} N, a_{\mu}=n^{v} \nabla_{\nu} n_{\mu}=(1 / N) h_{\mu}{ }^{\nu} \nabla_{\nu} N=(1 / N) D_{\mu} N$, where we denote by $D$ the covariant derivative operator induced on the time slices by the interior metric $h$, furthermore $\chi_{\mu \nu}=\frac{1}{2} \mathcal{L}_{n} h_{\mu \nu}$, the second fundamental form induced by $g$ on the time slices, and the coefficients $\gamma_{\mu}^{\nu}{ }_{\rho}=\Gamma_{\lambda^{\eta}}{ }_{\pi} h_{\mu}^{\lambda} h_{\eta}^{\nu} h_{\rho}^{\pi}$, which represent the covariant derivative $D$ such that the components $\gamma_{i}{ }^{j}{ }_{k}$ are the Christoffel symbols of the metric $h_{i j}$.

Finally we need the decomposition of the conformal Weyl tensor

$$
C_{\mu \nu \lambda \rho}=2\left\{l_{\mu[\lambda} E_{\rho] \nu}-l_{\nu[\lambda} E_{\rho] \mu}-n_{[\lambda} B_{\rho] \tau} \epsilon_{\mu \nu}^{\tau}-n_{[\mu} B_{\nu] \tau} \epsilon_{\lambda \rho}^{\tau}\right\}
$$

in terms of its $n$-electric part $E_{\tau \sigma}=C_{\mu \nu \lambda \rho} h^{\mu}{ }_{\tau} n^{\nu} h_{\sigma}^{\lambda} n^{\rho}$ and its $n$-magnetic part $B_{\tau \sigma}=$ $C_{\mu \nu \lambda \rho}^{*} h_{\tau}^{\mu} n^{\nu} h_{\sigma}^{\lambda} n^{\rho}$. Here we use the notation $l_{\mu \nu}=h_{\mu \nu}+n_{\mu} n_{\nu}$ and, with $\epsilon_{\mu \nu \lambda \rho}$ being the antisymmetric tensor satisfying $\epsilon_{0123}=\left|\operatorname{det}\left(g_{\mu \nu}\right)\right|^{\frac{1}{2}}=N\left\{\operatorname{det}\left(h_{i j}\right)\right\}^{\frac{1}{2}}$, we write $\epsilon_{\tau \sigma \eta}=\epsilon_{\mu \nu \lambda \rho} n^{\mu} h^{\nu}{ }_{\tau} h^{\lambda}{ }_{\sigma} h^{\rho}{ }_{\eta}$ and $C_{\mu \nu \lambda \rho}^{*}=\frac{1}{2} C_{\mu \nu \alpha \beta} \epsilon_{\lambda \rho}^{\alpha \beta}$. Using (3.8)-(3.10) we can express equations (3.3), (3.4) in terms of the 44 functions

$$
N, \quad S^{i}, \quad h_{i j}, \quad \gamma_{i}^{j}{ }_{k}, \quad \chi_{j k}, \quad E_{j k}, \quad B_{j k} .
$$




\section{The Bianchi equation}

The investigation of the Bianchi equation has a long history in general relativity. Its importance in the analysis of the long-time behaviour of solutions to the Einstein equations and its geometrical and interpretational interest in the study of gravitational radiation was perhaps recognized for the first time by Sachs [27] and demonstrated extensively by Newman and Penrose [24]. The system (3.5)-(3.7) provides in fact the tensorial basis of their spin frame formalism. Penrose pointed out the covariant transformation law of the Bianchi equation under conformal rescalings [25]. Trying to exploit this property in the context of global and semi-global existence theorems, I realized that the Bianchi equation implies hyperbolic equations and leads to energy estimates in terms of the Bel-Robinson tensor $[11,12,15]$. Later this fact was used in a more elaborate way by Christodoulou and Klainerman [4] in their work on the global nonlinear stability of Minkowski space.

If the connection coefficients are considered as given, the Bianchi equation (3.4) represents an overdetermined system of 16 linear equations for the 10 independent components of the conformal Weyl tensor. For our purpose the most interesting observation about that system is the fact that we can extract from it symmetric hyperbolic systems of propagation equations. In the spin frame formalism (3.4) reads

$$
\nabla^{f}{ }_{c^{\prime}} \Psi_{a b c f}=0
$$

where $\Psi_{a b c d}$ is the symmetric spinor field which represents the conformal Weyl tensor. Introducing the directional derivative operators

$$
\begin{array}{ll}
P=\nabla_{00^{\prime}}+\nabla_{11^{\prime}}=\sqrt{2} \nabla_{e_{0}}, & \mathcal{D}_{01}=\frac{1}{2}\left(\nabla_{00^{\prime}}-\nabla_{11^{\prime}}\right), \\
\mathcal{D}_{00}=-\nabla_{01^{\prime}}, & \mathcal{D}_{11}=\nabla_{10^{\prime}},
\end{array}
$$

we find that (4.1) is equivalent to the system consisting of the 'constraint equations' $\mathcal{D}^{a b} \Psi_{a b c d}=0$ and the propagation equations

$$
P \Psi_{a b c d}-2 \mathcal{D}_{(d}{ }^{f} \Psi_{a b c) f}=0
$$

which after a trivial transformation form a symmetric hyperbolic system [15]. It may be remarked that the operators introduced above arise naturally in the projection formalism based on the timelike vector field $\sqrt{2} e_{0}$ which leads to the space spinor formalism. Of course, the equations above can always be rewritten in terms of real fields, but their analysis is done most conveniently in terms of the symmetric spinor field. Still other symmetric hyperbolic systems can be extracted from (4.1), some of which are particularly well adapted to situations where data are partly prescribed on null [11,12] or timelike hypersurfaces [16].

In the ADM representation, the Bianchi equation (3.4) splits into the constraints

$$
D^{i} E_{i j}-B_{i k} \chi^{i}{ }_{l} \epsilon^{k l}{ }_{j}=0, \quad D^{i} B_{i j}+E_{i k} \chi^{i}{ }_{l} \epsilon^{k l}{ }_{j}=0,
$$

and the propagation equations

$$
\begin{aligned}
& \frac{1}{N}\left(\partial_{t}-\mathcal{L}_{S}\right) E_{i j}+D_{k} B_{l(i} \epsilon_{j)}{ }^{k l}-3 E^{k}{ }_{(i} \chi_{j) k}+\chi_{k}{ }^{k} E_{i j}-\epsilon_{i}{ }^{k l} E_{k m} \chi_{l n} \epsilon_{j}{ }^{m n} \\
& +2 a_{k} B_{l(i} \epsilon_{j)}{ }^{k l}=0, \\
& \frac{1}{N}\left(\partial_{t}-\mathcal{L}_{S}\right) B_{i j}-D_{k} E_{l(i} \epsilon_{j)}{ }^{k l}-3 B^{k}{ }_{(i} \chi_{j) k}+\chi_{k}{ }^{k} B_{i j}-\epsilon_{i}{ }^{k l} B_{k m} \chi_{l n} \epsilon_{j}{ }^{m n} \\
& -2 a_{k} E_{l(i} \epsilon_{j)}^{k l}=0 .
\end{aligned}
$$


Provided $N>0$ and $h_{j k}$ is positive definite, the system consisting of these two propagation equations satisfies the conditions of symmetric hyperbolicity if they are contracted with $h^{k(i} h^{j) l}$ and written in the form

$$
\begin{aligned}
& h^{k(i} h^{j) l} \frac{1}{N}\left(\partial_{t}-\mathcal{L}_{S}\right) E_{i j}+\frac{1}{2}\left(\epsilon^{i j(k} h^{l) m}+\epsilon^{i m(k} h^{l) j}\right) D_{i} B_{j m}+\cdots=0, \\
& h^{k(i} h^{j) l} \frac{1}{N}\left(\partial_{t}-\mathcal{L}_{S}\right) B_{i j}-\frac{1}{2}\left(\epsilon^{i j(k} h^{l) m}+\epsilon^{i m(k} h^{l) j}\right) D_{i} E_{j m}+\cdots=0 .
\end{aligned}
$$

Since the conformal Weyl tensor obeys hyperbolic equations, we are left with the analysis of (3.3) or equations (3.5), (3.6). Before discussing these systems we should observe the interesting structure of the characteristics of the propagation equations considered above. This is directly related to concepts of gravitational radiation which have been discussed in studies of the asymptotic behaviour of gravitational fields (cf [25, 27]). Only equations (4.4), (4.5) will be discussed, the situation is similar for (4.2).

For a given solution $g_{\mu \nu}$, a covector $\xi \neq 0$ is by definition characteristic for the system (4.4), (4.5) iff the linear map defined by the principal symbol at $\xi$ has non-trivial kernel, i.e. iff there is a non-trivial solution $\left(E_{i j}, B_{k l}\right)$ to

$$
n^{\mu} \xi_{\mu} E_{i j}-B_{m(i} \epsilon_{j)}^{m n} \xi_{n}=0, \quad n^{\mu} \xi_{\mu} B_{k l}+E_{p(k} \epsilon_{l)}^{p q} \xi_{q}=0 .
$$

Case $n^{\mu} \xi_{\mu}=0$. There are non-trivial solutions of the form

$$
B_{i j}=a\left(|\xi|^{2} h_{i j}-3 \xi_{i} \xi_{j}\right), \quad E_{i j}=c\left(|\xi|^{2} h_{i j}-3 \xi_{i} \xi_{j}\right)
$$

with real numbers $a, c$. We use the notation $|\xi|=\left(h^{i j} \xi_{i} \xi_{j}\right)^{\frac{1}{2}}$.

Case $k^{\mu \nu} \xi_{\mu} \xi_{v} \equiv\left(-n^{\mu} n^{v}+\frac{1}{4} h^{\mu \nu}\right) \xi_{\mu} \xi_{v}=0$. There are non-trivial solutions of the form

$$
B_{i j}=2 \xi_{(i} \tau_{j)}, \quad E_{i j}=\frac{1}{n^{\mu} \xi_{\mu}} \xi_{(i} \epsilon_{j)}{ }^{k l} \tau_{k} \xi_{l}
$$

with arbitrary $\tau_{j}$ satisfying $\xi^{j} \tau_{j}=0$. These are in fact two families of solutions which are distinguished by the sign of $n^{\mu} \xi_{\mu}$.

Case $g^{\mu v} \xi_{\mu} \xi_{v}=0$. There are non-trivial solutions satisfying

$$
\xi^{i} B_{i j}=0, \quad E_{i j}=\frac{1}{n^{\mu} \xi_{\mu}} B_{k(i} \epsilon_{j)}{ }^{k l} \xi_{l} .
$$

Again these define two 2-parameter families of solutions distinguished by the sign of $n^{\mu} \xi_{\mu}$. There are no further characteristics.

It follows that the characteristic polynomial of the system (4.4), (4.5) has the form $c\left(n^{\mu} \xi_{\mu}\right)^{k}\left(g^{\mu v} \xi_{\mu} \xi_{v}\right)^{j}\left(k^{\mu v} \xi_{\mu} \xi_{v}\right)^{l}$ with constant $c \neq 0$ and positive integers $k, j, l$. Thus the characteristics of the system are real and they are null hypersurfaces with respect to $g_{\mu \nu}$ or they are timelike and either tangent to $n$ or to the timelike cone $\left\{k_{\mu \nu} t^{\mu} t^{\nu}=0\right\}$ where $k_{\mu \nu}=-n_{\mu} n_{\nu}+4 h_{\mu \nu}$ such that $k^{\mu \nu} k_{\nu \rho}=\delta^{\mu}{ }_{\rho}$.

Denote by $\xi^{L}, L=0,1,2,3,4$, non-vanishing covectors at a given point $p \in M$ satisfying $n^{\mu} \xi_{\mu}^{L}=\left(1-\frac{1}{2} L\right)\left|\xi^{L}\right|$. By the previous discussion, all characteristic covectors at $p$ are of this type. For further discussion choose an orthonormal frame $e_{k}$ at $p$ with $e_{0}=n$ and fix the scaling and the spatial components of the covectors by the condition 
$h^{\mu \nu} \xi^{L}{ }_{v}=e^{\mu}{ }_{3}$. With $e_{k}$ we associate the pseudo-orthonormal frame $e_{a a^{\prime}}, a, a^{\prime}=0,1$ given by

$$
\begin{array}{ll}
e_{00^{\prime}}=\frac{1}{\sqrt{2}}\left(e_{0}+e_{3}\right), & e_{11^{\prime}}=\frac{1}{\sqrt{2}}\left(e_{0}-e_{3}\right), \\
e_{01^{\prime}}=\frac{1}{\sqrt{2}}\left(e_{1}+\mathrm{i} e_{2}\right), & e_{10^{\prime}}=\frac{1}{\sqrt{2}}\left(e_{1}-\mathrm{i} e_{2}\right)
\end{array}
$$

and assume a spin frame field $\iota_{a}, a=0,1$, such that $e_{a a^{\prime}}=\iota_{a} \bar{\iota}_{a^{\prime}}$. Suppose the Weyl spinor field is given in terms of such a spin frame and set, as usual, $\Psi_{L}=\Psi_{a b c d}$ for $L=a+b+c+d$. Then

$$
\begin{array}{ll}
\Psi_{0}=C_{\mu \nu \lambda \rho} e^{\mu}{ }_{00^{\prime}} e^{\nu}{ }_{01^{\prime}} e^{\lambda}{ }_{00^{\prime}} e^{\rho}{ }_{01^{\prime}}, & \Psi_{1}=C_{\mu \nu \lambda \rho} e^{\mu}{ }_{00^{\prime}} e^{\nu}{ }_{01^{\prime}} e^{\lambda}{ }_{00^{\prime}} e^{\rho}{ }_{11^{\prime}}, \\
\Psi_{2}=C_{\mu \nu \lambda \rho} e^{\mu}{ }_{00^{\prime}} e^{\nu}{ }_{01^{\prime}} e^{\lambda}{ }_{10^{\prime}} e^{\rho}{ }_{11^{\prime}}, & \\
\Psi_{3}=C_{\mu \nu \lambda \rho} e^{\mu}{ }_{00^{\prime}} e^{\nu}{ }_{11^{\prime}} e^{\lambda}{ }_{10^{\prime}} e^{\rho}{ }_{11^{\prime}}, & \Psi_{4}=C_{\mu \nu \lambda \rho} e^{\mu}{ }_{11^{\prime}} e^{\nu}{ }_{10^{\prime}} e^{\lambda}{ }_{11^{\prime}} e^{\rho}{ }_{10^{\prime}}
\end{array}
$$

and the discussion above can be rephrased by saying that for the characteristic covector $\xi^{L}$ the principal symbol has non-trivial kernel generated by $\Psi=\left(\Psi_{0}, \Psi_{1}, \Psi_{2}, \Psi_{3}, \Psi_{4}\right)$ with $\Psi_{K}=0$ for $K \neq L$ and arbitrary component $\Psi_{L}$.

The relationship between the type of characteristic and the fields in the kernel of the principal symbol map indicated above is important in the study of the propagation properties of the reduced equations. Because they are of particular interest, we add a few remarks on the characteristics determined by covectors of the type $\xi^{4}$. Suppose that the frame $e_{k}$ extends to a smooth frame field on some open neighbourhood of $p$ such that $e_{0}=n$ and let $N$ be a null hypersurface in this neighbourhood such that the null vector field $e_{00}$ is tangent to $N$. Then the characteristic covector field $g_{\mu \nu} e^{v}{ }_{00^{\prime}}$ is smooth and coincides at $p$ with $\xi_{\mu}^{4}$. On the given background we consider linear perturbations of the Weyl tensor obeying an equation whose principal part coincides with that of the symmetric hyperbolic system considered above for the conformal Weyl tensor. Following the discussion of the propagation of discontinuities in [5, p 618ff] we assume that the perturbation is a distributional solution which is continuous across $N$ with some of its first derivatives suffering jumps but having continuous limits on $N$ from the right and the left which are differentiable in directions tangent to $N$. It follows from the discussion above that the jumps occur only in the symmetric, transverse, trace-free components of $E_{i j}$ and $B_{i j}$ which are given by (4.6) with $n^{\mu} \xi_{\mu}=n^{\mu} g_{\mu \nu} e^{v} 0^{\prime}$. Loosely speaking, we could say that these components, also given by $\Psi_{4}$, contain the 'high-frequency' parts of perturbations of the conformal Weyl tensor which travel along the characteristic $N$. The two components of (4.6) correspond to the two polarization states of gravitational waves.

Now consider again solutions to the nonlinear reduced equations. Then the local consideration above may be supplemented by the observation of a somewhat global nature that in the case where $N$ extends smoothly to null infinity, the function $\Psi_{4}$, after suitable rescaling with an affine parameter on the null generators on $N$, approaches the radiation field on null infinity (cf [25]). Thus the discussion of the characteristics of the propagation equations (4.4), (4.5) should not only be of interest in the local numerical analysis, in particular near lightlike horizons, but also in the interpretation of numerical data concerning questions of gravitational radiation.

We remark that the function $\Psi_{0}$ has an analogous meaning with respect to null hypersurfaces coming in from past null infinity. On $N$ the function $\Psi_{0}$ represents the null datum for the field equations. The characteristics associated with $n^{\mu}$ and $k^{\mu \nu}$ arise by 
extracting the determined system (4.4), (4.5) from the overdetermined system (3.4). They depend on the chosen time slicing and have no invariant meaning; but they may be of interest in the numerical treatment of the equations.

\section{Reductions in the orthonormal frame representation}

Since the Bianchi equation implies symmetric hyperbolic equations for the conformal Weyl tensor, we are left with the analysis of the structure equations (3.5), (3.6). Three different ways of obtaining hyperbolic reduced equations will be shown. We shall only indicate the type of gauge condition and discuss the hyperbolic reduced equations. How the initial data for the reduced equations are prepared and how it follows that the gauge conditions and constraints are preserved under the evolution in the various cases follows from the discussions in $[13,15,16]$ and will not be repeated here.

\subsection{Gauge conditions based on wave equations}

Suppose a coordinate system $x^{\mu}$ and a frame $e_{k}$ have been chosen. Since for fixed index $\mu$ the differential $\mathrm{d} x^{\mu}$ is represented in the frame $e_{k}$ by the coefficients $e_{k}^{\mu}$, the coordinate gauge source functions are given by $f^{\mu}=-\nabla^{k} e^{\mu}{ }_{k}$. We characterize the additional freedom introduced by the frame field in terms of the 'frame gauge source functions' $f^{i}{ }_{j}=\nabla^{k} \Gamma_{k}{ }^{i}{ }_{j}$ where the 'covariant derivative' is defined here by the expression which is obtained for a tensor field which has the components $\Gamma_{k}{ }_{j}$ in the given frame. In a similar way as before we find that with any coordinate system and any frame field there are associated gauge source functions $f^{\mu}, f^{i}{ }_{j}$ and that for arbitrarily given functions $f^{\mu}\left(x^{\nu}\right), f^{i}{ }_{j}\left(x^{\nu}\right)$ coordinates and frame fields can locally be found for which these functions take the meaning of gauge source functions as discussed above [13]. By identifying these gauge source functions there are various ways to arrive at hyperbolic propagation equations.

If we recall how the Lorentz gauge is used in Maxwell's equations to derive a system of wave equations for the potential and if we compare with (3.5) and (3.6), we see that prescribing the gauge source functions above allows one to derive systems of wave equations for the frame coefficients $e^{\mu}{ }_{l}$ and for the connection coefficients $\Gamma_{l}{ }_{j}$. In the case of (3.6), which has been considered recently in [26], equations for the $\Gamma_{l}{ }^{i}{ }_{j}$ are obtained in which the conformal Weyl tensor no longer occurs because of (3.7). It suffices therefore to couple these equations to suitable equations for the frame coefficients. Finally, we recall that it has been known for a long time that the Bianchi equation implies a system of wave equations for the conformal Weyl tensor. Since we consider the information on the conformal Weyl tensor as particularly valuable and do not see any advantage in going to systems of higher order, we shall discuss in the following only reductions following from the structure equations and the Bianchi equation without taking further derivatives.

We note that in spinor notation the first structure equation is equivalent to

$$
\nabla_{a} f^{\prime} e_{b f^{\prime}}^{\mu}+\epsilon_{a b} f^{\mu}=0
$$

and its complex conjugate, and the second structure equation is equivalent to

$$
\begin{aligned}
& 2 \nabla_{c}{ }^{f^{\prime}} \Gamma_{d f^{\prime}}{ }^{a b}+\Gamma_{(c}{ }^{e^{\prime} f(a} \Gamma_{d) e^{\prime}}{ }^{b)}{ }_{f}+\epsilon_{c d} f^{a b}=-2 \phi^{a b}{ }_{c d}, \\
& 2 \nabla^{f}{ }_{c^{\prime}} \Gamma_{f d^{\prime}}{ }^{a b}+\Gamma^{e}{ }_{\left(c^{\prime}\right.}{ }^{f^{\prime}(a} \Gamma_{\left.d^{\prime}\right) e^{b)}}{ }_{f}+\epsilon_{c^{\prime} d^{\prime}} f^{a b}=0,
\end{aligned}
$$

where $e^{\mu}{ }_{a a^{\prime}}$ and $\Gamma_{a a^{\prime} b c}$ denote the frame and the connection coefficients, respectively, and the frame gauge source function is represented by $f^{a b}=f^{a}{ }_{a^{\prime}} b a^{\prime}$. These equations have 
been used in [13] to deduce propagation equations which combine, e.g. with (4.2), to yield symmetric hyperbolic propagations equations. We shall not reproduce the system here but note the remarkable feature that the gauge source functions enter the equations above in undifferentiated form. It appears to be the only system with this property. It allows us to prescribe gauge source functions which depend not only on the coordinates but also on the unknowns $e^{\mu}{ }_{k}, \Gamma_{i}{ }_{k}{ }_{k}, C^{i}{ }_{j k l}$ without changing the principal part of the system. The question of how this could be used to steer the evolution of the gauge has not been studied yet.

\subsection{Geometric gauge conditions}

Suppose we are given, as in the ADM formalism, a nowhere vanishing 'time flow vector field' $T$ and a time function $t=x^{0}$ such that $\langle\mathrm{d} t, T\rangle=1$. We write $T=T^{k} e_{k}$ and assume that $T^{0}>0$. We assume, furthermore, coordinates $x^{\alpha}$ with $\alpha=1,2,3$, such that $T^{k} e^{\mu}{ }_{k}=T^{\mu}=\delta^{\mu}{ }_{0}$, whence $T=\partial_{t}$. The propagation of the time slices is thus determined in terms of the functions $T^{k}$. We note that in the generality above, the functions $T^{k}$ were first considered as gauge source functions in [26]. The evolution of the frame field is fixed in terms of the functions $T^{k}{ }_{j}=T^{l} \Gamma_{l}{ }_{j}{ }_{j}$ since $\nabla_{T} e_{j}=T^{k}{ }_{j} e_{k}$.

It turns out that we can consider the functions $T^{k}\left(t, x^{\alpha}\right), T^{k}{ }_{j}\left(t, x^{\alpha}\right)$ as freely specifiable gauge source functions. The gauge conditions are then expressed by

$$
e^{\mu}{ }_{0}=\frac{1}{T^{0}}\left(\delta^{\mu}{ }_{0}-T^{a} e^{\mu}{ }_{a}\right), \quad \Gamma_{0}{ }_{k}=\frac{1}{T^{0}}\left(T^{j}{ }_{k}-T^{a} \Gamma_{a}{ }^{j}{ }_{k}\right),
$$

where $a=1,2,3$ and summation is implied. Observing this in the structure equations and taking into account that $\Psi_{a b c d}$ and $C^{i}{ }_{j k l}$ are related by a linear transformation with constant coefficients, we deduce for the remaining 40 unknowns $e^{\mu}{ }_{a}, \Gamma_{a}{ }_{j}^{i}, C^{i}{ }_{j k l}$ the symmetric hyperbolic propagation equations

$$
\begin{aligned}
& \partial_{t} e^{\mu}{ }_{a}=\left(T_{a}^{l}-T_{, v}^{l} e^{v}{ }_{a}-\Gamma_{a j}^{l} T^{j}\right) e^{\mu}{ }_{l}, \\
& \partial_{t} \Gamma_{a}{ }^{i}{ }_{k}=T_{k, \mu}^{i} e^{\mu}{ }_{a}+\Gamma_{a l}{ }_{l} T^{l}{ }_{k}-\Gamma_{a k}{ }_{k} T^{i}{ }_{l}+\left(T^{l}{ }_{a}-T^{l}{ }_{, \nu} e^{\nu}{ }_{a}-\Gamma_{a}{ }^{l} T^{p}\right) \Gamma_{l k}{ }_{k}+C^{i}{ }_{k l a} T^{l}, \\
& P \Psi_{a b c d}-2 \mathcal{D}_{(d}^{f} \Psi_{a b c) f}=0 .
\end{aligned}
$$

The functions $T^{k}\left(t, x^{\alpha}\right)$ with $T^{0}>0$ can be prescribed here completely freely. However, the cone of characteristic rays contains, besides the cone determined by the subsystem arising from the Bianchi equation, the direction $\partial_{t}$. To ensure causal propagation of the gauge (and to avoid certain other possible problems) we require $0 \leqslant g\left(\partial_{t}, \partial_{t}\right)=\eta_{k j} T^{k} T^{j}$.

Nevertheless, it is remarkable that we have free control of the causal nature of the time flow vector field. Since its coefficients may be given explicitly, it may be much easier in this gauge to study the influence of the gauge source functions on the evolution of the time slicing. The particular choice $T^{k}{ }_{j}=0$, i.e. parallel transport of the frame in the direction of $T$, leads to a considerable simplification of the equations above, but the freedom to specify the evolution of the frame may be used to adapt it to the particular situation under investigation. An interesting way to prescribe $T$ is to choose it timelike in the interior and null 'far out'. This allows a smooth transition between the standard Cauchy problem and the characteristic Cauchy problem ('Cauchy-characteristic matching').

\subsection{Mixed gauge conditions}

Assume again a vector field $T$ and coordinates $x^{\mu}$ as above, but write $N=T^{0}$, and set $N_{a}=e_{a}(N)$. In distinction from the previous case we assume now that $e_{0}$ is the future 
directed unit normal to the slices $\{t=$ constant $\}$ and the frame is Fermi propagated in the direction of $e_{0}$.

We find that we can prescribe $T^{a}\left(t, x^{\alpha}\right), a=1,2,3$, and $f^{0}\left(t, x^{\alpha}\right)=-\nabla_{k} \nabla^{k} t$ as gauge source functions. We note that this gauge, in which the time slicing is determined by a wave equation, is similar to the one employed in [3]. The gauge conditions translate into

$$
\begin{array}{llrl}
e^{\mu}{ }_{0} & =\frac{1}{N}\left(\delta^{\mu}{ }_{0}-T^{a} e^{\mu}{ }_{a}\right), & e^{0}{ }_{a}=0, & a=1,2,3, \\
\Gamma_{0}{ }^{0}{ }_{a} & =\frac{1}{N} N_{a}, & \Gamma_{0}{ }^{a}{ }_{b} & =0 .
\end{array}
$$

This leaves us with the 38 unknowns $N, N_{a}, e^{\alpha}{ }_{a}, \Gamma_{a}{ }^{b}{ }_{c}, \chi_{a b}, C^{i}{ }_{j k l}, a, b, c=1,2,3$, where $\Gamma_{a}{ }^{b}{ }_{c}$ contains the information about the Levi-Civita connection of the interior metric on the slices $\{t=$ constant $\}$ and $\chi_{a b}=-\Gamma_{a}{ }^{0}{ }_{b}$ is their extrinsic curvature.

Commutating derivatives of $N$ and observing the structure equations, we derive the following symmetric hyperbolic propagation equations:

$\partial_{t} e^{\alpha}{ }_{a}=\left\{T^{c}\left(\Gamma_{c}{ }^{b}{ }_{a}-\Gamma_{a}{ }^{b}{ }_{c}\right)-e_{a}\left(T^{b}\right)\right\} e_{b}^{\alpha}$,

$\Gamma_{a}{ }^{b}{ }_{c, \mu} e^{\mu}{ }_{0}=-\chi_{a}{ }^{d} \Gamma_{d}{ }^{b}{ }_{c}+\frac{1}{N}\left(e_{c}(N) \chi_{a}{ }^{b}-\eta^{b d} e_{d}(N) \chi_{a c}\right)+C^{b}{ }_{c 0 a}$

$N_{, \mu} e_{0}^{\mu}=N \chi+N^{2} f^{0}$,

$\frac{1}{N} N_{a, \mu} e_{0}^{\mu}-D^{c} \chi_{c a}=\frac{2}{N} \chi N_{a}+\frac{1}{N} \chi_{a}{ }^{c} N_{c}+3 N_{a} f^{0}+N e_{a}\left(f^{0}\right)$,

$N \chi_{a a, \mu} e^{\mu}{ }_{0}+D_{a} N_{a}=-N \chi_{a}{ }^{b} \chi_{a b}-N C_{a 0 a}^{0}, \quad a=1,2,3$,

$2 N \chi_{a c, \mu} e^{\mu}{ }_{0}+D_{a} N_{c}+D_{c} N_{a}=-2 N \chi_{a}{ }^{b} \chi_{c b}-2 N C_{c 0 a}^{0}, \quad a \neq c$,

$P \phi_{a b c d}-2 \mathcal{D}_{(d}{ }^{f} \phi_{a b c) f}=0$.

Besides various timelike or null directions, the cone of characteristic rays contains again the direction $\partial_{t}$. For causal propagation, we thus have to ensure that $N^{2} \geqslant \delta_{a b} T^{a} T^{b}$ in the evolution. This causality condition is different from the previous one because $N$ is no longer freely specifiable. Apart from this requirement, the gauge source functions can be given arbitrarily. The symmetric hyperbolic system above supplies in a most economic and direct way all the interesting information: the four-dimensional metric, given in terms of the frame coefficients, the four-dimensional connection, the conformal Weyl tensor, and the geometry of the time slices as well as their embedding. It may also be noted that the algebraic structures of the equations given in this and the previous section are very simple as compared to that of many other reduced systems.

We end the discussion of hyperbolic reductions in orthonormal frame representations with two remarks. There is no obvious way how the equations considered above can be rewritten in 'flux conserving form'. If this form turns out to be as important in relativity as advertised in some of the articles on hyperbolic reductions (cf [1]) and as it has proved to be in the case of some other quasilinear systems, this may be a serious drawback.

One of the most important reasons to consider the Bianchi equation is its behaviour under rescalings of the metric. By introducing a few more unknowns (so that we end up, depending on the choice of gauge, with about 55 unknowns) we can represent Einstein's equations in a conformal metric and still get hyperbolic equations. Doing this we trade a few unknowns against an infinite number of grid points or, in other words, we can 'calculate an 
infinite world on a finite grid'. It may well be that this property of the Bianchi equation can compete with the numerical advantages offered in other systems by the flux conserving form. Furthermore, the conformal equations allow one to make use of the elegant interpretational framework based on null infinity.

\section{Hyperbolic reductions in the ADM representation}

In the ADM representation the propagational part of the Einstein equations is usually expressed by the ADM equations

$$
\begin{aligned}
& \partial_{t} h_{i j}-\mathcal{L}_{S} h_{i j}=2 N \chi_{i j}, \\
& \frac{1}{N}\left(\partial_{t} \chi_{k l}-\mathcal{L}_{S} \chi_{k l}-D_{k} D_{l} N\right)=-{ }^{3} R_{k l}-\chi_{i}{ }^{i} \chi_{k l}+2 \chi_{i k} \chi_{l}{ }^{i} .
\end{aligned}
$$

Traditionally the fundamental forms $h_{i j}, \chi_{k l}$ are considered here as the basic unknowns and the lapse function $N$ and the components $S^{i}$ of the shift vector field are considered as free gauge source functions. Because of the terms of second order occuring in the Ricci tensor on the right-hand side, it is obvious that the system, as it stands, does not satisfy any known hyperbolicity condition. Consequently, a direct analytic investigation of this system is an outstanding problem.

Nevertheless, the system is close to being hyperbolic. Substituting $\chi_{i j}$ from equation (6.1) into equation (6.2) we get an equation of second order for $h_{i j}$ of the form

$$
\begin{aligned}
\frac{1}{2 N^{2}}\left\{\partial_{t}^{2} h_{i j}\right. & \left.-S^{k} \partial_{k} \partial_{t} h_{i j}-S^{k} \partial_{t} \partial_{k} h_{i j}+S^{k} S^{l} \partial_{k} \partial_{l} h_{i j}\right\}+{ }^{3} R_{i j} \\
& =\text { terms of lower order in } h_{i j} .
\end{aligned}
$$

For a given solution $g_{\mu \nu}$, the principal symbol of this system defines, for any covector $\xi_{\mu}$, a linear map $k_{i j} \rightarrow A_{i j}^{k l}(\xi) k_{k l}$ with

$$
\begin{aligned}
A_{i j}^{k l}(\xi) k_{k l}= & \frac{1}{2 N^{2}}\left(\xi_{0}^{2} k_{i j}-2 \xi_{0} S^{k} \xi_{k} k_{i j}+S^{k} \xi_{k} S^{l} \xi_{l} k_{i j}\right) \\
& -\frac{1}{2} h^{k l}\left(\xi_{k} \xi_{l} k_{i j}+\xi_{i} \xi_{j} k_{k l}-\xi_{i} \xi_{l} k_{k j}-\xi_{k} \xi_{j} k_{i l}\right) \\
= & \frac{1}{2}\left\{-g^{\mu \nu} \xi_{\mu} \xi_{v} k_{i j}-\left(\frac{1}{2} h^{k l} k_{k l} \xi_{i}-\xi^{k} k_{k i}\right) \xi_{j}-\left(\frac{1}{2} h^{k l} k_{k l} \xi_{j}-\xi^{k} k_{k j}\right) \xi_{i}\right\}
\end{aligned}
$$

where we set $\xi^{k}=h^{k l} \xi_{l}$.

A covector $\xi_{\mu} \neq 0$ is characteristic for the PDE above iff for this covector the map above has non-trivial kernel. If $\xi_{\mu} \neq 0$ satisfies

$$
g^{\mu v} \xi_{\mu} \xi_{v}=0
$$

the principal symbol has non-trivial kernel consisting of the solutions to $\frac{1}{2} h^{k l} k_{k l} \xi_{i}=\xi^{k} k_{k i}$. Secondly, if $g^{\mu v} \xi_{\mu} \xi_{v} \neq 0$ and $k_{i j} \neq 0$ is in the kernel, then necessarily $k_{i j}=\xi_{i} \eta_{j}+\eta_{i} \xi_{j}$ with some covector $\eta$. Inserting this into the equation $A_{i j}^{k l}(\xi) k_{k l}=0$ we get the characteristic condition

$$
n^{\mu} \xi_{\mu}=0 .
$$

Conversely, if this condition is satisfied, $k_{i j}=\xi_{i} \eta_{j}+\eta_{i} \xi_{j}$ is in the kernel of the symbol map for arbitrary $\eta$. Thus the characteristic polynomial is of the form $c\left(n^{\mu} \xi_{\mu}\right)^{k}\left(g^{\nu \rho} \xi_{\nu} \xi_{\rho}\right)^{j}$ with constant $c \neq 0$ and positive integers $k, j$ satisfying $k+2 j=12$. All characteristics are real and either null with respect to $g_{\mu \nu}$ or timelike and tangent to $n$. Obviously the system is not strictly hyperbolic. 
Since (6.3) satisfies this weakest concept of hyperbolicity, it may be surmised that the $\mathrm{ADM}$ equations represent only a half-way reduction of equation (6.3) to a system of first order which possibly satisfies a stronger requirement of hyperbolicity. In [18] the ADM equations are rewritten as a system of first order for 30 unknowns in which lapse and shift are considered as free gauge source functions. The system given by the authors can be rewritten in symmetric hyperbolic form. This is most remarkable, since it clarifies for the first time the sense in which the ADM equations can be considered as hyperbolic and it shows also for the first time that lapse and shift can be prescribed freely.

In [1] the ADM equations are rewritten as a system of first order for 49 unknowns, but the analysis of hyperbolicity given there does not go beyond the analysis given above. In [3] a symmetric hyperbolic system of first order for 58 unknowns is derived. It is based, however, not directly on the ADM equations but on derivatives of these equations and of the momentum constraints. Consequently some of the unknowns are of second order.

Instead of taking the ADM equations as a starting point, we shall combine (6.1) with equations implied by (3.1), (3.3) (cf (6.16), (6.17)) to derive a hyperbolic system including (4.4), (4.5). For this purpose the identity $\gamma_{i}{ }^{i}{ }_{k}=\frac{1}{2} h^{i j} \partial_{k} h_{i j}=\partial_{k} \log (\sqrt{h})$ with $h=\operatorname{det}\left(h_{i k}\right)$ is used. Two slightly different systems will be considered below; there may well be other possibilities for deducing hyperbolic systems involving the Bianchi equation.

In the first case the function $q=\log (N / \sqrt{h})$ and the components $S^{i}$ of the shift vector field are considered as freely specifiable gauge source functions. We obtain the following system for the 40 unknowns $h_{i j}, \gamma_{j}{ }^{i}, \chi_{i j}, E_{i j}, B_{i j}$ :

$$
\begin{aligned}
& \frac{1}{N}\left(\partial_{t}-\mathcal{L}_{S}\right) h_{i j}=2 \chi_{i j}, \\
& \frac{1}{N} h_{i l}\left(\partial_{t}-\mathcal{L}_{S}\right) \gamma_{j}^{l}{ }_{k}-D_{i} \chi_{j k}-a_{j} \chi_{i k}+a_{i} \chi_{j k}-a_{k} \chi_{i j}-\frac{1}{N} \partial_{j} \partial_{k} S^{l} h_{l i}=2 \epsilon_{i(k}^{l} B_{j) l},
\end{aligned}
$$

$$
\frac{1}{N}\left(\partial_{t}-\mathcal{L}_{S}\right) \chi_{i j}-\partial_{k} \gamma_{i}{ }^{k}{ }_{j}-\partial_{i} \partial_{j} q+\gamma_{i}{ }_{j} \partial_{k} q-a_{i} a_{j}+\gamma_{i}{ }^{k}{ }_{m} \gamma_{k}{ }^{m}{ }_{j}-\chi_{k}{ }^{k} \chi_{i j}=-2 E_{i j}
$$

$$
\begin{aligned}
\frac{1}{N}\left(\partial_{t}-\mathcal{L}_{S}\right) E_{i j} & +D_{k} B_{l(i} \epsilon_{j)}{ }^{k l}-3 E^{k}{ }_{(i} \chi_{j) k}+\chi_{k}{ }^{k} E_{i j}-\epsilon_{i}{ }^{k l} E_{k m} \chi_{l n} \epsilon_{j}{ }^{m n} \\
& +2 a_{k} B_{l(i} \epsilon_{j)}{ }^{k l}=0
\end{aligned}
$$

$$
\frac{1}{N}\left(\partial_{t}-\mathcal{L}_{S}\right) B_{i j}-D_{k} E_{l(i} \epsilon_{j)}{ }^{k l}-3 B^{k}{ }_{(i} \chi_{j) k}+\chi_{k}{ }^{k} B_{i j}-\epsilon_{i}{ }^{k l} B_{k m} \chi_{l n} \epsilon_{j}{ }^{m n}
$$

$$
-2 a_{k} E_{l(i} \epsilon_{j)}^{k l}=0
$$

We set here $N=\sqrt{h} e^{q}$ and $a_{i}=\partial_{i} q+\gamma_{k}{ }^{k}{ }_{i}$ and use the notation

$$
\mathcal{L}_{S} \gamma_{j}{ }_{k}{ }_{k}=S^{l} \partial_{l} \gamma_{j}{ }^{i}{ }_{k}+\gamma_{l}{ }_{k}{ }_{k} \partial_{j} S^{l}-\gamma_{j k}{ }_{k} \partial_{l} S^{i}+\gamma_{j}{ }_{l}{ }_{l} \partial_{k} S^{l} .
$$

If $N>0$ and $h_{i j}$ is positive definite the system is symmetric hyperbolic.

In the second case we consider the functions $S^{i}$ and $s=-N^{2} f^{0}-\partial_{k} S^{k}$, with $f^{0}=-\nabla_{\mu} \nabla^{\mu} t$, as freely specifiable gauge source functions. Notice that this includes as a particular choice the harmonic time slicing condition $f^{0}=0$, i.e. $s=-\partial_{k} S^{k}$.

In addition to the unknowns above we introduce the 10 unknowns $q, q_{i}=\partial_{i} q$, $q_{j i}=\partial_{j} \partial_{i} q$. Using (3.9) and (6.4), we find that they satisfy the equations

$$
\left(\partial_{t}-\mathcal{L}_{S}\right) q=s,
$$




$$
\begin{aligned}
& \left(\partial_{t}-\mathcal{L}_{S}\right) q_{i}=\partial_{i} s, \\
& \left(\partial_{t}-\mathcal{L}_{S}\right) q_{j i}=\partial_{j} \partial_{i} S^{k} q_{k}+\partial_{j} \partial_{i} s .
\end{aligned}
$$

The system (6.4)-(6.11), with $\partial_{i} q, \partial_{j} \partial_{i} q$ replaced by $q_{i}, q_{j i}$, respectively, is again symmetric hyperbolic under the proviso given above.

Besides those discussed in the section on the Bianchi equation, we point out further interesting properties of the systems above. We have obtained systems which supply in a most economic way all the information on four-dimensional spacetime, including the relevant fields of second order, as well as the inner and embedding geometry of the time slicing. Furthermore, both systems of propagation equations can easily be written in the 'flux conserving' form $\partial_{t} u+\partial_{i} F^{i}(x, u)=S(x, u)$. Finally, after simple extensions, the systems can be considered as subsystems of the conformal field equations. The interest of the latter in numerical investigations has to some extent been demonstrated in [21, 22].

\subsection{Propagation of the constraints}

Since neither (6.4)-(6.8) nor (6.4)-(6.11) has been considered in the literature before, we shall outline the arguments which show that solutions to data satisfying the constraints do indeed satisfy Einstein's field equations. We shall only consider the first system in detail, the argument being similar in the second case.

The initial data have to satisfy the constraint equations

$$
\begin{gathered}
\partial_{k} \gamma_{l}{ }_{j}-\partial_{l} \gamma_{k}{ }^{i}{ }_{j}+\gamma_{k}{ }^{i}{ }_{m} \gamma_{l}{ }^{m}{ }_{j}-\gamma_{l}{ }_{m} \gamma_{k}{ }^{m}{ }_{j}+\chi_{k}^{i} \chi_{j l}-\chi_{l}^{i} \chi_{j k} \\
={ }^{3} R^{i}{ }_{j k l}+\chi^{i}{ }_{k} \chi_{j l}-\chi^{i}{ }_{l} \chi_{j k} \\
=2\left\{h^{i}{ }_{[k} E_{l] j}-h_{j[k} E_{l]}{ }^{i}\right\}, \\
D_{k} \chi_{j i}-D_{j} \chi_{k i}=-B_{i l} \epsilon_{k j}^{l}
\end{gathered}
$$

implied by (3.1), (3.3). We denote by ${ }^{3} R^{i}{ }_{j k l}$ the curvature tensor of $h_{i j}$ and by ${ }^{3} R$ its Ricci scalar. The data for (6.4)-(6.8) are obtained from standard initial data $h_{i j}, \chi_{i j}$ on the three-dimensional initial hypersurface $S=\{t=0\}$ as follows. We assume the smooth Riemannian metric $h_{i j}$ and the smooth symmetric tensor field $\chi_{i j}$ to be given such that they satisfy the vacuum constraints

$$
{ }^{3} R+\left(\chi_{k}^{k}\right)^{2}-\chi_{i j} \chi^{i j}=0, \quad D^{k} \chi_{k j}-D_{j} \chi_{k}^{k}=0
$$

which are obtained by contractions of (6.12), (6.13). The connection coefficients $\gamma_{i}{ }_{k}{ }_{k}$ are of course the Christoffel symbols derived from $h_{i j}$. We use (6.12), contracted over the indices $i$ and $k$, and (6.13) to obtain

$E_{l j}=\partial_{i} \gamma_{l}^{i}{ }_{j}-\partial_{l} \partial_{j} \log (\sqrt{h})+\gamma_{l}{ }_{j}{ }_{j} \partial_{m} \log (\sqrt{h})-\gamma_{l m}{ }^{i} \gamma_{i}{ }^{m}{ }_{j}+\chi_{i}{ }^{i} \chi_{j l}-\chi_{i l} \chi_{j}^{i}$,

and $B_{i j}=-\epsilon_{j}^{k l} D_{k} \chi_{l i}$ on $S$. Then $h_{i j}, \chi_{i j}, E_{i j}, B_{i j}$ do not only satisfy (6.13) but also (6.12) because the space is of dimension three. Moreover, they satisfy the constraints (4.3). Choosing a function $N>0$ on $S$, we can determine the function $q$ on $S$. We assume $q$ to be extended to a smooth function $q=q(t, x)$ with $(t, x) \in M=\mathbb{R} \times S$, and we assume a smooth vector field $S^{\mu}=S^{\mu}(t, x)$ with $S^{0}=0$ as given on $M$. Then all quantities in the propagation equations can be expressed in terms of the unknowns and the fields $q$, $S^{i}$, the derivative operator $D$ being of course defined by the connection coefficients $\gamma_{i}{ }^{j} k$ provided by the solution. Since the system is symmetric hyperbolic there exists in a certain neighbourhood $U$ of $S \equiv S \times\{0\}$ in $M$ a smooth solution of (6.4)-(6.8) which takes the 
given data on $S$ and for which $h_{i j}$ is positive definite. From $q$ and $h_{i j}$ we can determine the smooth positive function $N$ on $U$ which allows us to determine the metric $g_{\mu \nu}$ on $U$. We denote the domain of dependence of $S$ in $U$ with respect to this metric by $V$.

We show that $g_{\mu \nu}$ satisfies Einstein's vacuum field equations on $V$. Since equation (6.4) is part of the system of propagation equations, it follows that the field $\chi_{i j}$ supplied by the solution indeed represents the second fundamental form induced by $g_{\mu \nu}$ on the slices $\{t=$ constant $\}$. At this stage it is not clear, however, whether the $\gamma_{i}{ }^{j}{ }_{k}$ supplied by the solution coincide with the Christoffel symbols of the metric $h_{i j}$. Though the connections corresponding to the $\Gamma_{\mu}{ }^{\nu} \rho$ and the $\gamma_{i}{ }^{j}{ }_{k}$ are symmetric because the symmetry is demanded explicitly for the latter, we do not know at this stage whether they are metric for $g_{\mu \nu}$ and $h_{i k}$, respectively. We denote the Christoffel symbols derived from $h_{i j}$ by $\bar{\gamma}_{i}{ }^{j}{ }_{k}$ and the associated covariant derivative operator by $\bar{D}$. If $\gamma_{i}{ }^{j}{ }_{k}$ coincides with the Christoffel symbols for $h_{i j}$, the coefficients (3.9) are the Christoffel symbols for $g_{\mu \nu}$. We therefore show that the 'zero quantities'

$$
z=\left(\gamma_{i}{ }^{j}{ }_{k}-\bar{\gamma}_{i}{ }^{j}, \Delta^{\mu}{ }_{\nu \lambda \rho}=R_{\nu \lambda \rho}^{\mu}-C^{\mu}{ }_{\nu \lambda \rho}, H_{\nu \lambda \rho}=\nabla_{\mu} C^{\mu}{ }_{\nu \lambda \rho}\right)
$$

vanish on $V$. In the following we shall denote by $L(z)$, sometimes with indices attached to $L$, expressions which are linear in the zero quantities. Examples of such expressions, which will occur in the following calculations, are given by $\gamma_{i}{ }^{i}{ }_{k}-\partial_{k} \log (\sqrt{h})=\gamma_{i}{ }^{i}{ }_{k}-\bar{\gamma}_{i}{ }^{i}$, $D_{i} h_{j k}=D_{i} h_{j k}-\bar{D}_{i} h_{j k}$, etc.

We have the decomposition

$$
\begin{aligned}
\Delta_{\nu \lambda \rho}^{\mu}=\delta^{\mu}{ }_{\nu \lambda \rho} & +n^{\mu}\left(n_{\lambda} \Delta_{v \rho}-n_{\rho} \Delta_{v \lambda}\right) \\
& +n_{\nu}\left(n_{\lambda} \delta_{\rho}^{\mu}-n_{\rho} \delta_{\lambda}^{\mu}\right)-n_{\lambda} \Delta_{\nu \rho}^{\mu}+n_{\rho} \Delta_{\nu \lambda}^{\mu}-n^{\mu} \delta_{\nu \lambda \rho}-n_{\nu} \delta^{* \mu}{ }_{\lambda \rho}
\end{aligned}
$$

with

$$
\begin{array}{ll}
\delta_{\nu \lambda \rho}^{\mu}=h_{\alpha}^{\mu} \Delta_{\beta \gamma \delta}^{\alpha} h_{\nu}^{\beta} h_{\lambda}^{\gamma} h_{\rho}^{\delta}, & \Delta_{\nu \rho}=n_{\alpha} \Delta_{\beta \gamma \delta}^{\alpha} h_{\nu}^{\beta} n^{\gamma} h_{\rho}^{\delta}, \\
\delta_{\rho}^{\mu}=h^{\mu}{ }_{\alpha} \Delta_{\beta \gamma \delta}^{\alpha} n^{\beta} n^{\gamma} h_{\rho}^{\delta}, & \Delta^{\mu}{ }_{\nu \rho}=h^{\mu}{ }_{\alpha} \Delta_{\beta \gamma \delta}^{\alpha} h^{\beta}{ }_{\nu} n^{\gamma} h_{\rho}^{\delta}, \\
\delta_{\nu \lambda \rho}=n_{\alpha} \Delta_{\beta \gamma \delta}^{\alpha} h^{\beta}{ }_{\nu} h^{\gamma}{ }_{\lambda} h^{\delta}, & \delta^{* \mu}{ }_{\lambda \rho}=h^{\mu}{ }_{\alpha} \Delta^{\alpha}{ }_{\beta \gamma \delta} n^{\beta} h^{\gamma}{ }_{\lambda} h^{\delta}{ }_{\rho} .
\end{array}
$$

With this notation the propagation equations (6.5), (6.6) are equivalent to

$$
\begin{aligned}
& h_{\mu \lambda} \Delta_{\nu \rho}^{\lambda}+\delta_{\nu \mu \rho}+h_{\mu \lambda} D_{\rho} \chi_{\nu}^{\lambda}-D_{\rho} \chi_{\mu \nu}=0, \\
& \Delta_{\nu \rho}+\delta^{\mu}{ }_{\nu \mu \rho}+D_{\rho} \gamma_{\mu}{ }^{\mu}{ }_{\nu}-D_{\rho} D_{\nu} \log (\sqrt{h})=0,
\end{aligned}
$$

respectively.

Observing that the right dual of a tensor possessing the symmetries of a conformal Weyl tensor coincides with its left dual and using the fact that $R^{\mu}{ }_{\nu \lambda \rho}$ satisfies the Bianchi identity, we get

$$
\epsilon_{\nu}{ }^{\alpha \beta \gamma} \nabla_{\alpha} \Delta_{\lambda \beta \gamma}^{\mu}=-\epsilon_{\lambda}^{\mu \alpha \beta} H_{\nu \alpha \beta}+L(z)=L^{\prime}(z) .
$$

In the decomposition (6.15) we take into account that we solved the propagation equations (6.16), (6.17) and insert the resulting expression on the left-hand side of (6.18). Evaluation of

$$
-n_{\rho} h_{\nu}^{\alpha} \epsilon_{\tau}^{\beta \gamma \delta} h_{\mu}^{\tau} \nabla_{\beta} \Delta^{\rho}{ }_{\alpha \gamma \delta}, \quad-h_{\mu \rho} h_{\nu}^{\alpha} \epsilon_{\tau}^{\beta \gamma \delta} h_{\mu}^{\tau} \nabla_{\beta} \Delta_{\alpha \gamma \delta}^{\rho},
$$

gives equations

$$
\begin{aligned}
& \frac{1}{N}\left(\partial_{t}-\mathcal{L}_{S}\right) \tilde{\delta}_{i j}+D_{k} \tilde{\delta}^{k}{ }_{i j}=L_{i j}(z), \\
& \frac{1}{N} h_{l k}\left(\partial_{t}-\mathcal{L}_{S}\right) \tilde{\delta}^{k}{ }_{i j}+D_{l} \tilde{\delta}_{i j}=L_{l i j}(z),
\end{aligned}
$$


respectively, where we used the notation

$$
\tilde{\delta}_{\mu \nu}=\frac{1}{2} \tilde{\delta}_{\mu \lambda \rho} \epsilon^{\lambda \rho}{ }_{\nu}, \quad \tilde{\delta}^{\kappa}{ }_{\mu \nu}=\frac{1}{2} \tilde{\delta}^{\kappa}{ }_{\mu \lambda \rho} \epsilon^{\lambda \rho}{ }_{\nu} .
$$

We note that at some stage in the somewhat lengthy calculation the relation

$$
D^{\mu} \tilde{\delta}_{\nu \mu}=D^{\mu} B_{\mu \nu}+\epsilon_{\nu}{ }^{\alpha \beta} E_{\beta \kappa} \chi_{\alpha}{ }^{\kappa}+L_{v}(z)=L_{\nu}^{\prime}(z)
$$

has been used. From (6.4) the equation

$$
\begin{aligned}
\frac{1}{N} h_{i l}\left(\partial_{t}-\mathcal{L}_{S}\right) & \bar{\gamma}_{j}^{l}{ }_{k}-\bar{D}_{k} \chi_{j i}-\bar{D}_{j} \chi_{k i}+\bar{D}_{i} \chi_{j k} \\
& -a_{j} \chi_{i k}+a_{i} \chi_{j k}-a_{k} \chi_{i j}+\frac{1}{N} \partial_{j} \partial_{k} S^{l} h_{l i}=0
\end{aligned}
$$

follows by direct calculation. By suitable subtractions of (6.5), $\delta_{j k i}$, and $\delta_{k j i}$ we arrive at an equation of the form

$$
\frac{1}{N}\left(\partial_{t}-\mathcal{L}_{S}\right)\left(\gamma_{j k}^{l}-\bar{\gamma}_{j k}^{l}\right)=L_{j k}^{\prime l}(z)
$$

Using the (manifest) symmetries of the tensor $C^{\mu}{ }_{\nu \lambda \rho}$ we get

$$
\nabla^{\nu} H_{\nu \lambda \rho}=\nabla^{\nu} \nabla_{\mu} C_{\nu \lambda \rho}^{\mu}=C_{\alpha \beta \lambda \rho} \Delta_{\mu}^{\mu \alpha \beta}-C^{\alpha \beta}{ }_{\tau[\lambda} \Delta_{\rho] \alpha \beta}^{\tau} .
$$

Again by the symmetries of the field $C^{\mu}{ }_{\nu \lambda \rho}$, we find the decomposition

$$
\begin{gathered}
H_{\nu \lambda \rho}=q_{\nu \tau} \epsilon_{\lambda \rho}^{\tau}-n_{\lambda} p_{\nu \rho}+n_{\rho} p_{\nu \lambda}+\frac{1}{2}\left(n_{\rho} q_{\tau} \epsilon_{\nu \lambda}^{\tau}-n_{\lambda} q_{\tau} \epsilon_{\nu \rho}^{\tau}-2 n_{\nu} q_{\tau} \epsilon_{\lambda \rho}^{\tau}\right) \\
+\frac{1}{2}\left(3 n_{\nu} n_{\lambda}+h_{\nu \lambda}\right) p_{\rho}-\frac{1}{2}\left(3 n_{\nu} n_{\rho}+h_{\nu \rho}\right) p_{\lambda}
\end{gathered}
$$

with

$$
\begin{array}{ll}
q_{\nu \tau}=\frac{1}{2} H_{\alpha \beta \gamma} h^{\alpha}{ }_{(\nu} \epsilon_{\tau}{ }^{\beta \gamma}, & p_{\nu \rho}=H_{\alpha \beta \gamma} h^{\alpha}{ }_{(\nu} n^{\beta} h^{\gamma}{ }_{\rho)}, \\
q_{\tau}=\frac{1}{2} H_{\alpha \lambda \rho} n^{\alpha} \epsilon_{\tau}{ }^{\lambda \rho}, & p_{\rho}=H_{\alpha \beta \gamma} n^{\alpha} n^{\beta} h_{\rho}^{\gamma} .
\end{array}
$$

With this notation the propagation equations (6.7), (6.8) are equivalent to

$$
q_{\nu \tau}=0, \quad p_{\mu \rho}=0,
$$

and the constraint equations (4.3) are satisfied if and only if $q_{\tau}$ and $p_{\rho}$ vanish.

Using on the left-hand side of (6.22) the decomposition above with (6.23) we deduce the equations

$$
\begin{gathered}
3 n^{\lambda} \nabla_{\lambda} p_{\mu}+\epsilon_{\mu}^{\alpha \beta} D_{\alpha} q_{\beta}-3 n_{\mu} a^{\alpha} p_{\alpha}+3 \epsilon_{\mu}^{\alpha \beta} a_{\alpha} q_{\beta}-\chi_{\mu}{ }^{\alpha} p_{\alpha}+4 \chi_{\alpha}{ }^{\alpha} p_{\mu} \\
=-2\left(C_{\alpha \beta \lambda \rho} \Delta^{v \alpha \beta}{ }_{\nu}-C^{\alpha \beta}{ }_{\tau[\lambda} \Delta^{\tau}{ }_{\rho] \alpha \beta}\right) n^{\lambda} h^{\rho}{ }_{\mu}, \\
2 n^{\lambda} \nabla_{\lambda} q_{\mu}-\epsilon_{\mu}^{\alpha \beta} D_{\alpha} p_{\beta}-2 n_{\mu} a^{\alpha} q_{\alpha}-\chi_{\mu}{ }^{\alpha} q_{\alpha}+3 \chi_{\alpha}{ }^{\alpha} q_{\mu} \\
=-2\left(C_{\alpha \beta \lambda \rho} \Delta^{v \alpha \beta}{ }_{\nu}-C^{\alpha \beta}{ }_{\tau \lambda} \Delta^{\tau}{ }_{\rho \alpha \beta}\right) \epsilon^{\lambda \rho}{ }_{\mu},
\end{gathered}
$$

which imply equations of the form

$$
\begin{aligned}
& h^{i l} \frac{3}{N}\left(\partial_{t}-\mathcal{L}_{S}\right) p_{l}+\epsilon^{i k l} D_{k} q_{l}=L_{p}^{i}(z), \\
& h^{i l} \frac{2}{N}\left(\partial_{t}-\mathcal{L}_{S}\right) q_{l}-\epsilon^{i k l} D_{k} p_{l}=L_{q}^{i}(z) .
\end{aligned}
$$

The system of 'subsidiary equations' (6.19)-(6.21), (6.26), (6.27) is symmetric hyperbolic with characteristic polynomial $c\left(n^{\mu} \xi_{\mu}\right)^{k}\left(g^{\mu v} \xi_{\mu} \xi_{v}\right)^{j} l^{\mu v} \xi_{\mu} \xi_{v}$. Here $c$ is a non-vanishing constant, $k, j$ are positive integers, and $l^{\mu \nu}=-6 n^{\mu} n^{\nu}+h^{\mu \nu}$. It follows that the 
characteristics of this system are timelike or null with respect to $g_{\mu \nu}$. The zero quantities either vanish because the propagation equations are satisfied or they are linear functions of $\tilde{\delta}_{i j}, \tilde{\delta}^{k}{ }_{i j}, \gamma_{j k}{ }^{l}-\bar{\gamma}_{j k}{ }_{k}, p_{l}, q_{j}$. Furthermore the system of subsidiary equations is homogeneous while the data vanish on $S$. It follows that the zero quantities vanish on $V$.

\section{References}

[1] Bona C, Masso J, Seidel E and Stela J 1995 A new formalism for numerical relativity Phys. Rev. Lett. 75 600

[2] Choquet-Bruhat Y and York J W Jr 1980 The Cauchy problem General Relativity and Gravitation vol I, ed A Held (New York: Plenum)

[3] Choquet-Bruhat Y and York J W Jr 1995 Geometrical well posed system for the Einstein equations Preprint gr-qc/5906071

[4] Christodoulou D and Klainerman S 1993 The Global Nonlinear Stability of the Minkowski Space (Princeton, NJ: Princeton University Press)

[5] Courant R and Hilbert D 1962 Methods of Mathematical Physics vol II (New York: Interscience)

[6] De Donder T 1921 La gravifique einsteinienne (Paris: Gauthier-Villars)

[7] DeTurck D 1981 Existence of metrics with prescribed Ricci curvature: local theory Invent. Math. 65 179-207

[8] DeTurck D 1983 The Cauchy problem for Lorentz metrics with prescribed Ricci curvature Compositio Math. 48 327-49

[9] Fischer A E and Marsden J E 1972 The Einstein evolution equations as a first-order quasi-linear symmetric hyperbolic system: I Commun. Math. Phys. 28 1-38

[10] Fourès-Bruhat Y 1952 Théorèm d'existence pour certain systèmes d'équations aux dérivées partielles non linéaires Acta. Math. 88 141-225

[11] Friedrich H 1981 On the regular and the asymptotic characteristic initial value problem for Einstein's vacuum field equations Proc. R. Soc. A 375 169-84

[12] Friedrich H 1981 The asymptotic characteristic initial value problem for Einstein's vacuum field equations as an initial value problem for a first-order quasilinear symmetric hyperbolic system Proc. R. Soc. A 378 401-21

[13] Friedrich H 1985 On the hyperbolicity of Einstein's and other gauge field equations Commun. Math. Phys. $100525-43$

[14] Friedrich H 1986 On the existence of $n$-geodesically complete or future complete solutions of Einstein's field equations with smooth asymptotic structure Commun. Math. Phys. 107 587-609

[15] Friedrich H 1991 On the global existence and the asymptotic behaviour of solutions to the Einstein-MaxwellYang-Mills equations J. Diff. Geom. 34 275-345

[16] Friedrich H 1995 Einstein equations and conformal structure: existence of anti-de Sitter-type space-times J. Geom. Phys. 17 125-84

[17] Friedrichs K O 1954 Symmetric hyperbolic linear differential equations Commun. Pure Appl. Math. $7345-92$

[18] Frittelli S and O Reula 1994 On the Newtonian limit of general relativity Commun. Math. Phys. 166 221-35

[19] Graham C R and Lee J M 1991 Einstein metrics with prescribed conformal infinity on the ball Adv. Math. 87 186-225

[20] Hawking S W and Ellis G F R 1973 The Large-scale Structure of Spacetime (Cambridge: Cambridge University Press)

[21] Hübner P 1993 Numerische und analytische Untersuchungen von (singulären) asymptotisch flachen Raumzeiten mit konformen Techniken Thesis Universität München

[22] Hübner P 1996 Method for calculating the global structure of (singular) spacetimes Phys. Rev. D 53701

[23] Lanczos C 1922 Ein vereinfachendes Koordinatensystem für die Einsteinschen Gravitationsgleichungen Z. Phys. 23 537-9

[24] Newman E T and Penrose R 1992 An approach to gravitational radiation by a method of spin coefficients J. Math. Phys. 3 566-78

[25] Penrose R 1965 Zero rest-mass fields including gravitation: asymptotic behaviour Proc. R. Soc. A 284 159-203

[26] van Putten M H P M and Eardley D M 1996 Nonlinear wave equations for relativity Phys. Rev. D 53 3056-63

[27] Sachs R K 1961 Gravitational waves in general relativity: VI. The outgoing radiation condition Proc. $R$. Soc. A 264 309-38 